\title{
Vacuum Cleaner Artifact
}

National Cancer Institute

\section{Source}

National Cancer Institute. Vacuum Cleaner Artifact. NCI Thesaurus. Code C86988.

A magnetic field perturbation artifact that results in multiple high intensity rings. 